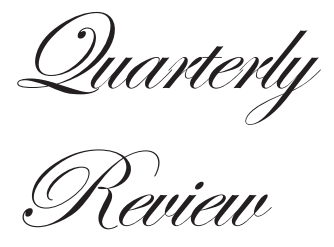

\title{
Diet, nutrient deficiency and chronic pancreatitis.
}

\author{
Gopalakrishna Rajesh ${ }^{1}$, Banavara Narasimhamurthy Girish ${ }^{2}$, Kannan \\ Vaidyanathan $^{3}$, Vallath Balakrishnan ${ }^{1}$
}

\section{ABSTRACT}

Departments of Gastroenterology ${ }^{1}$, Physiology $^{2} \&$ Biochemistry ${ }^{3}$

Amrita Institute of Medical

Sciences, Kochi,

AIMS Ponekkara P.O.

Cochin - 682041

Kerala, India

Correspondence:

Professor Vallath Balakrishnan

Email: vbalakrishnan@ aims.amrita.edu
The aetiopathogenesis of chronic pancreatitis $(\mathrm{CP})$ appears to be multifactorial with interplay of genetic and environmental factors such as alcohol, smoking and diet. Dietary factors in the form of a fat- and protein-rich diet are important cofactors in the aetiopathogenesis of alcoholic pancreatitis. Malnutrition used to be a hallmark in tropical pancreatitis. Presently, it appears that malnutrition is an effect rather than a causative factor for tropical pancreatitis; however, micronutrient deficiency could possibly be implicated in the aetiopathogenesis. The role of dietary toxins such as cassava is no longer considered an important risk factor; however, these patients are at a higher risk for defective detoxification of cyanogens. Decrease in levels of sulphur amino acids (SAAs), folate and zinc have been reported. Oxidative stress and antioxidant depletion are key pathogenetic mechanisms. The role of antioxidants in pain relief is an area of interest. Supplementation of fat-soluble vitamins and micronutrients is a cornerstone in medical management. Folate and zinc supplementation may be beneficial and is a focus of ongoing research. In the future, prophylaxis for $\mathrm{CP}$ by a daily micronutrient tablet among high-risk groups in endemic areas could prove to be a potentially important public health measure. However, further studies are required.

KEYWORDS: chronic pancreatitis, micronutrients, dietary toxins, alcoholic pancreatitis

\section{Introduction}

Chronic pancreatitis (CP) is defined as a continuing inflammatory disease of the pancreas characterized by irreversible morphological changes typically causing pain and/ or permanent loss of function. The role of dietary factors in the aetiopathogenesis of alcoholic CP and idiopathic chronic pancreatitis (ICP) including tropical chronic pancreatitis (TCP) is an area of ongoing interest. Most cases of TCP have been reported from the state of Kerala in India.

\section{Tropical chronic pancreatitis (TCP)}

TCP may be defined as a form of ICP seen in tropical Asian and African countries; characterized by abdominal pain, intraductal calculi and diabetes mellitus in young, non-alcoholic subjects. Zuidema from Indonesia first reported a series of 45 patients with pancreatic calcification with diabetes mellitus who were poor and consumed a protein- and calorie-deficient diet, and also had striking clinical features of malnutrition such as emaciation, parotidomegaly, hair and skin changes not unlike that of Kwashiorkor. ${ }^{1}$ This was followed by a series of reports of similar patients from various tropical countries in Asia (India, Bangladesh, Sri Lanka), Africa (Uganda, Nigeria, Zambia, Madagascar) and South America (Brazil) following which TCP came to be recognized as a distinct entity with unique clinical and epidemiological features different from that of ACP. The largest series from the south-western state of Kerala in India 
by Geevarghese et al.; reported that these patients typically had "pain in childhood, diabetes in adolescence and death during prime of life". ${ }^{2}$

TCP occurs usually in children or young adults and is characterized by recurrent abdominal pain, large pancreatic intraductal calculi, development of diabetes mellitus, steatorrhoea, malnutrition, and a high rate of development of pancreatic cancer. Recent reports suggest that the presentation is changing. ${ }^{3-6}$ The age of onset is getting older and the disease course seems to be milder. Overt steatorrhoea is unusual (except when exposed to dietary fat challenge). However, unique features remain the strong propensity to develop diabetes mellitus (well before exocrine failure), and marked calcifications in a grossly dilated main pancreatic duct. Better management of diabetes mellitus and its complications, and maldigestion have resulted in longer survival and better outcomes. Improvement in socioeconomic conditions, better healthcare facilities, improved nutrition and sanitation are other factors which could have impacted the disease characteristics.

\section{Dietary and nutrient factors in aetiopathogenesis of $\mathbf{C P}$}

\section{Alcoholic chronic pancreatitis (ACP)}

Usually, consumption of alcohol for several years is necessary to produce ACP; however, there is no threshold value below which the disease does not occur. In most patients, at least 5 years of alcohol intake exceeding $80 \mathrm{~g} /$ day is required before the development of CP. Only 5\%-15\% of alcoholics develop $\mathrm{CP}$ suggesting the role of some genetic factors or associated cofactor(s). Potential cofactors that have been proposed include a diet rich in fat and protein, a relative deficiency of antioxidants or trace elements, and smoking. Cessation of alcohol use after the onset of ACP appears to variably diminish the rate of progression to exocrine and endocrine insufficiency.

\section{Tropical chronic pancreatitis (TCP)}

Several hypotheses have been proposed; however, there is still a lack of definite understanding of the aetiopathogenesis of TCP. Malnutrition was initially suspected to play a causal role. TCP was thought to occur more commonly among poor and malnourished patients as this was indeed the characteristic epidemiological feature in most early reports. Pancreatic fibrosis was shown to develop in chronic protein-starved rats. However, recent studies suggest that malnutrition appeared to be an effect rather than the cause. ${ }^{7}$ TCP is now increasingly observed in the affluent and well-nourished patients and rarely even in the obese. The possibility of micronutrient deficiency contributing a predominant role is still an attractive proposition. ${ }^{8}$ Micronutrient deficiency could impact pancreatic function as some of these do appear to play a vital though often not well-characterized role in pancreatic function. Alternatively, micronutrient deficiency could be implicated in the production of oxidative stress which is well known to be implicated in the pathogenesis of $\mathrm{CP}$.

Initially, dietary toxins such as Manihot esculenta (cassava, tapioca), a tuber which contains varying amounts of cyanogen glycosides were implicated. Reports suggested an epidemiological association of cassava consumption and prevalence of TCP in Kerala. The hypothesis by McMillan and Geeverghese indicated an association between dietary cyanide and TCP. ${ }^{9}$ The postulated mechanism essentially was that hydrocyanic acid, liberated from cyanogen glycosides (linamarin and lotaustralin) of cassava or other foods by action of gastric hydrochloric acid produced pancreatic damage. However, TCP is reported from several parts of India and the world where cassava is not consumed; conversely TCP was found to be rare in many populations eating large quantities of cassava. The processing and preparation of cassava appears to play some modifying effects. A case-control study has also shown a lack of association between cassava and TCP. ${ }^{10}$ We have reported a significant reduction in rhodanese activity with concomitant decrease in sulphur amino acids (SAA) and antioxidants suggesting that patients with TCP may be at a higher risk for detoxification of cyanogens. ${ }^{11}$ However, we did not find any difference between consumers and nonconsumers of cassava.

\section{Review of nutrient factors}

\section{(1) Sulphur amino acids (SAAs)}

We have reported reductions in plasma amino acids particularly SAAs and branched chain amino acids in patients with $\mathrm{CP}{ }^{12}$ The emerging links between SAA and diseases of relevance to public health indicate that knowledge of the regulation of SAA metabolism is of growing importance. Homocysteine is a SAA having a highly reactive sulphur group, which is the reason for its reaction with other molecules. Its metabolism is intimately linked with the metabolism of folate, vitamin $B_{12}$ (cobalamin) and pyridoxine. Homocysteine occupies a branch point in the synthesis of methionine and cysteine and thereby glutathione. 
Remethylation of homocysteine to methionine occurs in most cells of the body; however, transsulphuration occurs only in the pancreas, liver, kidney and small intestine. Martensson and Bolin indicated disturbed metabolism of SAAs in chronic relapsing pancreatitis. ${ }^{13}$ The intermediate products of transmethylation pathway may have an influence on the development of pancreatitis.

A classic study by Farber and Popper showed that methionine supplementation prevents ethionine-induced pancreatitis in experimental animals. ${ }^{14}$ Subsequently, a study by Parsa et al. on rat pancreatic culture showed that methionine appears to be an obligatory requirement for the differentiation of the pancreatic acinar cells. ${ }^{15}$ This issue has been addressed more directly by Capdevila et al. showing that ethionine pretreatment inhibits amylase secretion from freshly isolated pancreatic acini. ${ }^{16}$ The same investigators showed that several inhibitors of methylation inhibit amylase secretion in vitro by AR42J cells (derived from a rat pancreatic acinar cell carcinoma).

In pancreas, Glycine N-methyltransferase (GNMT) is abundant principally in the pancreatic acinar cells. ${ }^{17}$ GNMT catalyses the methylation of glycine into sarcosine, coupled with conversion of the methyl donor, S-adenosylmethionine (AdoMet), into S-adenosylhomocysteine (AdoHcy). GNMT is believed to play a role in monitoring the AdoMet/AdoHcy ratio, and hence the cellular methylation capacity. Methionine deficiency may have adverse effects on pancreatic growth, differentiation and survival because SAAs are essential in a number of chemical reactions. Ida et al. noted that methionine antagonist (ethionine) in choline-deficient animals would provide a permissive environment for $\mathrm{CP}$ to progress towards pancreatic cancer. ${ }^{18}$

\section{(2) Folate}

We showed lower plasma methionine levels in two cohorts of patients with $\mathrm{CP}$ - one of TCP and the other of ACP as compared to healthy controls, which suggests that deficiency of methyl groups may be a factor in various forms of pancreatitis. Similarly, we have shown lower red cell glutathione levels in patients with TCP and ACP, indicating deficiency of thiol molecules. In addition, we have shown significantly higher levels of plasma total homocysteine in patients with $\mathrm{CP}$ than in healthy controls. Moreover, our study has shown that there is a deficiency of red cell folate in the majority of patients with $\mathrm{CP}$, more so in TCP; and that folate deficiency appeared to be the key factor in hyperhomocysteinaemia in patients with $\mathrm{CP} .{ }^{19}$ It is yet to be elucidated whether high plasma homocysteine levels have any pathogenetic implications through possible vascular damage in the pancreas, or they merely serve as a marker of the disease. ${ }^{20,21}$ Altered folate metabolism due to 5, 10methylenetetrahydrofolate reductase (MTHFR) polymorphisms could play a role in $\mathrm{CP}$.

A recent study on north Indian patients showed association of MTHFR (C667T) polymorphism in susceptibility to TCP. ${ }^{22}$ MTHFR is a key enzyme in folate metabolism. Since MTHFR is involved in methylation, inflammation and protection against oxidative stress, the process is especially important for pancreatic homeostasis. The altered enzyme activity could play a role in pancreatic injury. Previous studies have shown that C667T polymorphism increases the risk of pancreatic cancer.

There is emerging evidence for long-term studies on folate supplementation in CP. Apart from the liver, the pancreas is the other organ in the body which has the maximum folate content. ${ }^{23}$ Moreover, folate plays a crucial role in the integrity and normal physiology of the pancreas, ${ }^{24}$ which becomes altered in folate deficiency. Folate plays an important role in one carbon transfer involving remethylation of homocysteine to methionine, which is a precursor of AdoMet, a ubiquitous methyl donor. We have shown that patients with $\mathrm{CP}$ who had low levels of methionine also had low levels of red cell folate. There is epidemiological evidence linking folate deficiency to the development of pancreatic cancer. ${ }^{25}$ Aberrant DNA methylation could be a leading mechanism for the development of carcinogenesis in $\mathrm{CP}^{26}$

\section{(3) Zinc}

We have reported zinc deficiency in patients with $\mathrm{CP}$, and observed that zinc deficiency correlates with exocrine and endocrine insufficiency. ${ }^{27}$ We have reported a positive correlation of erythrocyte zinc and elastase 1 levels in patients with $\mathrm{CP}$, the latter being a measure of pancreatic exocrine function.

Pancreatic juice contains zinc in high concentrations as a constituent of metalloenzymes, such as carboxypeptidase and carbonic anhydrase. It has been suggested that the pancreas plays a major role in zinc homeostasis. ${ }^{28}$ Dutta et al. have shown increased zinc excretion in patients with pancreatic exocrine insufficiency. ${ }^{29}$

On the other hand, zinc deficiency could influence pancreatic function. Ultrastructural studies of pancreatic acinar cells in rats fed with a zinc-deficient diet showed the destruction 
of zymogen granules and lysosomes, ${ }^{30}$ indicating that zinc has a role in maintaining the structural integrity of pancreatic acinar cells. Rats with a marginal zinc deficiency showed morphological and functional alterations in the pancreas similar to those induced by alcohol. ${ }^{31}$ Zinc deficiency may play a role in ethanol-induced secretory alterations, because a poor intake of zinc is often associated with chronic alcoholism. ${ }^{32}$ Further, the increased activity of prolyl hydroxylase — an enzyme which takes part in the synthesis of collagen - in experimentally zincdepleted pancreatic tissue suggests that zinc deficiency is associated with pancreatic collagen deposition and may thus be implicated in pancreatic fibrosis. ${ }^{33}$

Zinc deficiency could be due to pancreatic exocrine insufficiency as evidenced by the direct correlation between erythrocyte zinc and pancreatic stool elastase 1 level. However, diabetes mellitus has been shown to be an independent risk factor for hypozincaemia. ${ }^{34,35} \mathrm{We}$ observed lower zinc levels in diabetic patients with $\mathrm{CP}$ as compared to non-diabetic patients with CP. This effect of diabetes mellitus on zinc levels was more marked in TCP as compared to ACP. ${ }^{27}$

We have observed that zinc deficiency appeared to affect oxidative stress in patients with $\mathrm{CP} .{ }^{36}$ Zinc deficiency can be a contributory factor in disease progression, via the reduction of free radical scavengers, increased oxidant stress and increased collagen deposition. Other possible effects of zinc deficiency could include an alteration in immune function.

\section{Dietary surveys}

The senior author has previously reported that patients with TCP had a poor intake of fat and protein as also the increased intake of cassava. ${ }^{37}$ Diets of patients with TCP and controls from Kerala were poorer as compared to patients with ACP and controls from France. A recent survey by our group showed that malnutrition remains an important problem in $\mathrm{CP}$; however, severe malnutrition is rare as compared to previous series. ${ }^{38}$ Energy (calorie) deprivation and micronutrient deficiency are the major nutritional issues.

\section{Nutritional status assessment}

We observed that faecal elastase 1 (marker of pancreatic exocrine insufficiency) correlated with faecal fat excretion and body mass index (BMI). ${ }^{39}$ We found evidence of pancreatic insufficiency in about half of the patients with normal BMI. Aggressive screening for malabsorption is a key strategy to detect early subclinical malnutrition. We observed that the use of anthropometric parameters such as triceps skin-fold thickness in conjunction with BMI was helpful. Subjective global assessment (SGA) was also found to be useful for assessing nutritional status.

\section{Dietary recommendations}

The major nutritional goals are to restore nutrient intake, digestion and absorption. Abstinence from alcohol helps improve nutrient intake. Adequate relief of pain results in increased dietary intake.

Restricting dietary fat minimizes pancreatic stimulation and decreases symptoms associated with steatorrhoea; hence it is a useful measure in medical management. Medium-chain triglycerides (MCTs) are useful dietary fat substitutes as they do not stimulate the pancreas. Shea et al. showed that a diet of MCTs resulted in a statistically significant improvement in pain as assessed by the visual analogue scale. ${ }^{40}$ They found that MCTs are useful in postprandial pain in a subset of patients with CP . In our survey, we found that coconut oil which is a widely used dietary component in Kerala is a good source of MCTs. ${ }^{38}$ A study from India showed that dietary counselling for a balanced home diet is as good as MCT-enriched food supplements. ${ }^{41}$

Fat-soluble vitamins A, D, E and $\mathrm{K}$ should be replaced in diet when deficient.

\section{Micronutrient supplementation}

Braganza et al. and Dormandy have made a case for nutrient supplementation in the prevention of pancreatitis in a susceptible population. ${ }^{42,43}$ Braganza et al. reported that ascorbate oxidation due to cooking practices prevalent in South India (Chennai) led to antioxidant depletion and could be likely implicated in oxidative stress and aetiopathogenesis of TCP. ${ }^{44}$ They have reported region-specific differences in micronutrient deficiency,e.g. ascorbic acid in Chennai (South India), selenium in Soweto (South Africa), etc.

From the various studies on antioxidants it appears that the recommended preparation would comprise 500-1000 mg vitamin C, 250-300 IU vitamin E, 500-800 ìg selenium, 2 g methionine, and 9000-10000 IU $\beta$-carotene.

A recent randomized controlled trial (RCT) from India involving 35 patients with $\mathrm{ACP}$ and 92 with ICP used antioxidant preparation with 600 ìg organic selenium, $0.54 \mathrm{~g}$ ascorbic acid, 
9000 IU â-carotene, 270 IU $\alpha$-tocopherol and $2 \mathrm{~g}$ methionine. This study reported significant reduction in the number of painful days per month $(7.4 \pm 6.8$ vs. $3.2 \pm 4, \mathrm{p}<0.001,95 \% \mathrm{CI}$ $2.07,6.23)$ and a significant reduction in the number of analgesic tablets per month $(10.5 \pm 11.8$ vs. $4.4 \pm 5.8, \mathrm{p}=0.001,95 \%$ CI 2.65 , 9.65). It also showed a significant reduction in the levels of oxidative stress. ${ }^{45}$

The composition of an ideal antioxidant preparation is still not clear. The case for folate and zinc supplementation is very strong. There is a need for further studies to determine the role of $\mathrm{B}_{6}$ supplementation. There are likely to be geographical differences, therefore antioxidant therapy should be tailored to region-specific needs.

\section{Conclusions}

The role of dietary and nutrient factors in the maintenance of normal pancreatic function and in the aetiopathogenensis of $\mathrm{CP}$ is an area of ongoing research. The management of nutrient deficiencies as a consequence of pancreatic endocrine and exocrine insufficiency is a key component in the medical management of these patients.

\section{References}

1. Zuidema PJ. Cirrhosis and disseminated calcification of the pancreas in patients with malnutrition. Trop Geogr Med. 1959;11:70-4.

2. Geeverghese PJ, Pitchumoni CS, Nair SR. Is protein malnutrition an initiating cause of pancreatic calcification? J Assoc Physicians India. 1969;17:417-19.

3. Balakrishnan V. Tropical chronic pancreatitis: a historical perspective. Gut. 2011;60:1441.

4. Balakrishnan V, Kumar H, Sudhindran S, Unnikrishnan AG (eds). Chronic Pancreatitis and Pancreatic Diabetes in India. Kochi: Indian Pancreatitis Study Group; 2005. Available at www.ipans.org (accessed on ?????).

5. Balakrishnan V, Nair P, Radhakrishnan L, Narayanan VA. Tropical pancreatitis-a distinct entity, or merely a type of chronic pancreatitis? Indian J Gastroenterol. 2006;25:74-81.

6. Balakrishnan V, Unnikrishnan AG, Thomas V, Choudhuri G, Veeraraju P, Singh SP, et al. Chronic pancreatitis. A prospective nationwide study of 1,086 subjects from India. JOP. 2008;2:593-600.

7. Midha S, Singh N, Sachdev V, Tandon RK, Joshi YK, Garg PK. Cause and effect relationship of malnutrition with idiopathic chronic pancreatitis: prospective case-control study. $J$ Gastroenterol Hepatol. 2008;23:1378-83.

8. Apte MV, Pirola RC, Wilson JS. Malnutrition as a cause of chronic pancreatitis: myth dispelled? J Gastroenterol Hepatol. 2008;23:1312-14.
9. McMillan DE, Geevarghese PJ. Dietary cyanide and tropical malnutrition diabetes. Diabetes Care. 1979;2:202-8.

10. Narendranathan M, Cheriyan A. Lack of association between cassava consumption and tropical pancreatitis syndrome. $J$ Gastroenterol Hepatol. 1994;9:282-5.

11. Girish BN, Rajesh G, Vaidyanathan K, Balakrishnan V. Assessment of cassava toxicity in patients with tropical pancreatitis. Trop Gastroenterol. 2011;32:112-6.

12. Girish BN, Rajesh G, Vaidyanathan K, Balakrishnan V. Alterations in plasma amino acid levels in chronic pancreatitis. JOP. 2011;12:11-8.

13. Martensson J, Bolin T. Sulfur amino acid metabolism in chronic relapsing pancreatitis. Am J Gastroenterol. 1986;81:1179-84.

14. Farber E, Popper H. Production of acute pancreatitis with ethionine and its prevention by methionine. Proc Soc Exp Biol Med. 1950;74:838-40.

15. Parsa I, Marsh WH, Fitzgerald PJ. Pancreas acinar cell differentiation. 3. Importance of methionine in differentiation of pancreas anlage in organ culture. Am J Pathol. 1970;59:1-22.

16. Capdevila A, Decha-Umphai W, Song KH, Borchardt RT, Wagner C. Pancreatic exocrine secretion is blocked by inhibitors of methylation. Arch Biochem Biophys. 1997;345:47-55.

17. Yeo EJ, Wagner C. Tissue distribution of glycine Nmethyltransferase, a major folate-binding protein of liver. Proc Natl Acad Sci USA. 1994;91:210-14.

18. Ida S, Ohmuraya M, Hirota M, Ozaki N, Hiramatsu S, Uehara $\mathrm{H}$, et al. Chronic pancreatitis in mice by treatment with cholinedeficient ethionine-supplemented diet. Exp Anim. 2010;59:421-9.

19. Girish BN, Vaidyanathan K, Rao AN, Rajesh G, Reshmi S, Balakrishnan V. Chronic pancreatitis is associated with hyperhomocysteinemia and derangements in transsulfuration and transmethylation pathways. Pancreas. 2010;39:e11-16.

20. Rajesh G, Girish BN, Vaidynathan K, Saumya M, Balakrishnan V. Folate deficiency in chronic pancreatitis. JOP. 2010;11:409-10.

21. Braganza JM, Odom N, McCloy RF, Ubbink JB. Homocysteine and chronic pancreatitis. Pancreas. 2010;39:1303; author reply 1304.

22. Singh S, Choudhuri G, Kumar R, Agarwal S. Association of 5, 10methylenetetrahydrofolate reductase $\mathrm{C} 677 \mathrm{~T}$ polymorphism in susceptibility to tropical chronic pancreatitis in north Indian population. Cell Mol Biol (Noisy-le-grand). 2012;58:122-7.

23. Balaghi M, Wagner C. Folate deficiency inhibits pancreatic amylase secretion in rats. Am J Clin Nutr. 1995;61:90-6.

24. Elseweidy M, Singh M. Folate deficiency and pancreatic acinar cell function. Proc Soc Exp Biol Med. 1984;177:247-52.

25. Larrson SC, Hakansson N, Giovannuccci E, Wolk A. Folate intake and pancreatic cancer incidence: a prospective study of Swedish women and men. J Natl Cancer Inst. 2006;98:407-13.

26. Larsson SC, Giovannucci E, Wolk A. Folate intake, MTHFR polymorphisms, and risk of esophageal, gastric and pancreatic cancer: a meta-analysis. Gastroenterology. 2006;131:1271-83.

27. Girish BN, Rajesh G, Vaidyanathan K, Balakrishnan V. Zinc status in chronic pancreatitis and its relation with exocrine and endocrine insufficiency. JOP. 2009;10:651-6.

28. McClain CJ. The pancreas and zinc homeostasis. J Lab Clin 
Med. 1990;116:275-6.

29. Dutta SK, Procaccino F, Aamodt R. Zinc metabolism in patients with exocrine pancreatic insufficiency. J Am Coll Nutr. 1998;17:556-63.

30. Koo SI, Turk DE. Effect of zinc deficiency on the ultrastructure of the pancreatic acinar cell and intestinal epithelium in the rat. $J$ Nutr. 1977;107:896-908.

31. Perez-Jimenez F, Bockman DE, Singh M. Pancreatic acinar cell function and morphology in rats fed zinc-deficient and marginal zinc-deficient diets. Gastroenterology. 1986;90:946-57.

32. McClain CJ, Su LC. Zinc deficiency in the alcoholic: a review. Alcohol Clin Exp Res. 1983;7:5-10.

33. Navarro S, Valderrama R, To-Figueras J, Giménez A, López JM, Campo E, et al. Role of zinc in the process of pancreatic fibrosis in chronic alcoholic pancreatitis. Pancreas. 1994;9:270-4.

34. Quilliot D, Dousset B, Guerci B, Dubois F, Drouin P, Ziegler O. Evidence that diabetes mellitus favors impaired metabolism of zinc, copper, and selenium in chronic pancreatitis. Pancreas. 2001;22:299-306.

35. Garg VK, Gupta R, Goyal RK. Hypozincemia in diabetes mellitus. J Assoc Physicians India. 1994;42:720-21.

36. Girish BN, Rajesh G, Vaidyanathan K, Balakrishnan V. Assessment of oxidative stress in chronic pancreatitis and its relation with zinc status. Indian J Gastroenterol. 2011;30:84-8.

37. Balakrishnan V, Sauniere JF, Hariharan M, Sarles H. Diet, pancreatic function, and chronic pancreatitis in south India and France. Pancreas. 1988;3:30-5.
38. Tinju J, Reshmi S, Rajesh G, Balakrishnan V. Anthropometric, biochemical, clinical and dietary assessment for malnutrition in south Indian patients with chronic pancreatitis. Trop Gastroenterol. 2010;31:285-90.

39. Girish BN, Rajesh G, Vaidyanathan K, Balakrishnan V. Fecal elastase 1 and acid steatocrit estimation in chronic pancreatitis. Indian J Gastroenterol. 2009;28:201-5.

40. Shea JC, Bishop MD, Parker EM, Gelrud A, Freedman SD. An enteral therapy containing medium-chain triglycerides and hydrolyzed peptides reduces postprandial pain associated with chronic pancreatitis. Pancreatology. 2003;3:36-40.

41. Singh S, Midha S, Singh N, Joshi YK, Garg PK. Dietary counseling versus dietary supplements for malnutrition in chronic pancreatitis: a randomized controlled trial. Clin Gastroenterol Hepatol. 2008;6:353-9.

42. Branagnaza JM, Dormandy TL. Micronutrient therapy for chronic pancreatitis: rationale and impact. JOP. 2010;11:99-112.

43. Braganza JM, Lee SH, McCloy RF, McMahon MJ. Chronic pancreatitis. Lancet. 2011;377:1184-97.

44. Braganza JM, Schofield D, Snehalatha C, Mohan V. Micronutrient antioxidant status in tropical compared with temperate-zone chronic pancreatitis. Scand J Gastroenterol. 1993;28:1098-104.

45. Bhardwaj P, Garg PK, Maulik SK, Saraya A, Tandon RK, Acharya SK. A randomized controlled trial of antioxidant supplementation for pain relief in patients with chronic pancreatitis. Gastroenterology. 2009;136:149-59. 